Pacific Journal of Mathematics

EXISTENCE OF A HOMOTOPY OPERATOR FOR SPENCER'S
SEQUENCE IN THE ANALYTIC CASE 


\section{EXISTENCE OF A HOMOTOPY OPERATOR FOR SPENCER'S SEQUENCE IN THE ANALYTIC CASE}

\section{Buttin}

According to E. Cartan's prolongation theorem, an analytic system of linear partial differential equations becomes an involutive system, after prolongation in a finite number of steps, and an involutive system has local solutions, by the Cartan-Kähler theorem.

Recently, a homological procedure has been developed, in terms of which the notion of involution is equivalent to the vanishing of a certain type of cohomology (so-called " $\delta$-cohomology"). Moreover, the local solvability of a linear system of partial differential equations has been shown by Quillen to be equivalent to the exactness, at degree one, of a certain resolution introduced originally by Spencer, which is canonically associated with the given system. The terms of the resolution are sheaves of germs of jet forms, i.e., differential forms with values in jet spaces.

The exactness of this resolution, providing a replacement for the Cartan-Kähler theorem in the linear case, in the analytic case is known. We shall have given another proof, based on the construction of a homotopy operator which is a natural generalization, to jet forms, of the well-known homotopy operator used in proving the Poincaré lemma for the exterior derivative $d$.

Some estimates will be necessary in order to study the existence of this operator, and we use here extensively the estimates obtained by Sweeney in [5] which are related to the bounds obtained earlier by L. Ehrenpreis, V. W. Guillemin and S. Sternberg in the paper [1].

1. Notation. Let $M$ be a $C^{\infty}$ manifold of real dimension $n$. Since everything we shall do is local, we shall always work in a neighborhood, $U$, of a reference point 0 of $M$. A coordinate $\left(x^{1}, x^{2}, \cdots, x^{n}\right)$, vanishing at 0 , will be chosen in $U$.

If $E$ is a $C^{\infty}$ bundle over $M, \underline{E}$ denotes the sheaf of germs of $C^{\infty}$ sections of $E$. But, for the sake of simplicity, and because $E$ can be supposed trivial over $U$, we shall in general use the same notation $E$ for both.

Also for the sake of simplicity, we shall use the following, now classical, condensed notations:

$p=\left(p_{1}, p_{2}, \cdots, p_{n}\right)$ is a multi-index of nonnegative integers $p_{i}$. 
The length of $p$, denoted $|p|$, is the sum $p_{1}+p_{2}+\cdots+p_{n}$.

$p$ ! is the product $p_{1} ! p_{2} ! \cdots p_{n}$ !

$x^{p}$ is the product $\left(x^{1}\right)^{p_{1}}\left(x^{2}\right)^{p_{2}} \cdots\left(x^{n}\right)^{p_{n}}$.

$\frac{\partial^{|p|} f}{\partial x^{p}}=\frac{\partial^{|p|} f}{\left(\partial x^{1}\right)^{p_{1}} \cdots\left(\partial x^{n}\right)^{p_{n}}}$ for the derivatives of the differentiable

functions in $U$.

We can define the sum $p+q$ of the multi-indices $p$ and $q$, by:

$$
p+q=\left(p_{1}+q_{1}, p_{2}+q_{\iota}, \cdots, p_{n}+q_{n}\right),
$$

and also their difference $p-q$, whenever $q_{1} \leqq p_{1}, \cdots, q_{n} \leqq p_{n}$, by

$$
p-q=\left(p_{1}-q_{1}, p_{2}-q_{2}, \cdots, p_{n}-q_{n}\right) \text {. }
$$

(The inequalities $q_{1} \leqq p_{1}, \cdots, q_{n} \leqq p_{n}$, may be condensed into $q \subset p$.) Finally, $p+1_{i}$ will denote the multi-index $\left(p_{1}, \cdots, p_{i}+1, \cdots, p_{n}\right)$.

2. Definitions and results required in the sequel. In this paragraph we shall recall, very briefly, some results which can be found in [3], [4(c)] and [5].

Let $E$ be a $C^{\infty}$ vector bundle over $M$, with fiber $\boldsymbol{R}^{m}$. We denote by $J^{\mu}(E)$ the vector bundle, over $M$, of all the jets of order $\mu$ of the germs of $C^{\infty}$ sections of $E$.

There is a natural projection $\pi^{\mu}: J^{\mu}(E) \rightarrow J^{\mu-1}(E)$. We can cover $J^{\mu}(E) \mid U$, if $E$ is trivial over $U$, by the coordinate $(x, \sigma)$, where $x$ is the coordinate $\left(x^{1}, \cdots, x^{n}\right)$ in $U$, and $\sigma=\left(\sigma_{p}^{j}\right), j=1,2, \cdots, m,|p| \leqq \mu$ is the coordinate in Hom $\left(\bigoplus_{0 \leqq \gamma \leqq \mu} S^{\gamma}\left(\boldsymbol{R}^{n}\right), \boldsymbol{R}^{m}\right)$ where $S^{\gamma}\left(\boldsymbol{R}^{n}\right)$ is the $\gamma$-fold symmetric product of $\boldsymbol{R}^{n}$.

From now on, whenever there is no possibility of confusion, we shall omit the subscript $j$, thus writing, for instance, $\sigma=\left(\sigma_{p}\right)$.

Finally, there is a map $j^{\mu}: \underline{E} \rightarrow \underline{J^{\mu}(E)}$ which is given in a local coordinate by

$$
f \longrightarrow \frac{\partial^{|p|} f}{\partial x^{p}} .
$$

Now let $E$ and $F$ be two $C^{\infty}$ vector bundles over $M$, with fibers $\boldsymbol{R}^{m}$ and $\boldsymbol{R}^{p}$, supposed trivial over $U$.

A linear differential operator $\mathscr{D}$, of order $\mu_{0}$, is a linear mapping

$$
\mathscr{D}: E \longrightarrow F
$$

locally given by

$$
\mathscr{D} f(x)=\sum_{|p| \leqq \mu_{0}} a^{p}(x) \frac{\partial^{|p|} f}{\partial x^{p}}(x)
$$


where the $a^{p}$ are $C^{\infty} l \times m$ matrix-valued functions.

We associate to $\mathscr{D}$ the (algebraic) linear operator:

$$
\mathscr{L}_{0}: J^{\mu_{0}}(E) \longrightarrow F
$$

defined by

$$
\mathscr{L}_{0}\left(\sigma_{p}\right)(x)=\sum_{|p| \leq \mu_{0}} a^{p}(x) \sigma_{p}(x)
$$

and we prolong $\mathscr{L}_{0}$, inductively, by differentiation, as follows:

$$
\mathscr{L}_{q}\left(\sigma_{p}\right)(x)=\sum_{\substack{p=r+s \\|r| \leq \mu_{0}, s \subset q}} a_{q}^{p}(x) \sigma_{p}(x) .
$$

Then $\mathscr{L}_{q+1_{i}}$ will be defined by

$$
\begin{aligned}
\mathscr{L}_{q+1_{i}}\left(\sigma_{p}\right)(x) & =\sum_{\substack{p=r+s \\
|r| \leqq \mu_{0}, s \subset q}}\left(\frac{\partial a_{q}^{p}(x)}{\partial x^{i}} \sigma_{p}(x)+a_{q}^{p}(x) \sigma_{p+1_{i}}(x)\right) \\
& =\sum_{\substack{p=r+s \\
|r| \leqq \mu_{0}, s \subset q+1_{i}}} a_{q+1_{i}}^{p}(x) \sigma_{p}(x) .
\end{aligned}
$$

Here $a_{0}^{p}(x)$ is naturally identified with $a^{p}(x)$.

The collection of the maps $\mathscr{L}_{q}$, for $|q|<\mu, \mu \geqq \mu_{0}$, which we shall denote by $\mathscr{L}^{\mu}$, is called the $\mu^{\text {th }}$ prolongation of $\mathscr{L}_{0}$. It maps $J^{\mu}(E)$ into $J^{\mu-\mu_{0}}(F)$ and makes the following diagram commute:

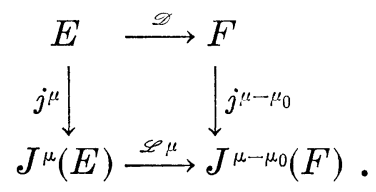

Let $\Sigma^{\mu}$ denote the kernel of $\mathscr{L}^{\mu}$.

We shall suppose, once and for all in this paper, that $\mathscr{D}$ is regular, that is:

(i) $\Sigma^{\mu}$ is a $C^{\infty}$ sub-bundle of $J^{\mu}(E)$.

(ii) The map $\Sigma^{\mu} \rightarrow \Sigma^{\mu-1}$ (induced by $\pi^{\mu}$ ) is surjective. This map will still be called $\pi^{\mu}$.

$\Sigma^{\infty}$ will signify the projective limit of $\Sigma^{\mu}$.

We finally introduce the following sheaves of germs of differential forms over $M$ :

$$
\Sigma^{\mu, r}=\Sigma^{\mu} \otimes \bigwedge^{r} T^{*}(M)
$$

where $T^{*}(M)$ is the cotangent bundle of $M$. Moreover, $\pi^{\mu}$ induces the projection, still denoted $\pi^{\mu}$,

$$
\pi^{\mu}: \Sigma^{\mu, r} \longrightarrow \Sigma^{\mu-1, r}
$$

and $\Sigma_{\mu-1}^{\mu, r}$ is by definition the kernel of $\pi^{\mu}$. An element $\sigma_{\mu-1}^{\mu, r}$ of $\Sigma_{\mu-1}^{\mu, r}$ 
is represented, in the local coordinate, by the components,

$$
\sigma_{p}=\sum_{j_{1} \leqq \cdots \leqq j_{r}} \sigma_{p_{1} \cdots p_{n}: j_{1} \cdots j_{r}} d x^{j_{1}} \wedge \cdots \wedge d x^{j_{r}}
$$

which we can write, more succinctly,

$$
\sigma_{p}=\Sigma_{j} \sigma_{p, J} d x^{J} .
$$

We now define the map $\delta$, namely the map

$$
\delta: \sum_{\mu-1}^{\mu, r} \longrightarrow \Sigma_{\mu-2}^{\mu-1, r+1},
$$

which is locally given by

$$
(\delta \sigma)_{p}=\sum_{i=1}^{n} d x^{i} \wedge \sigma_{p+1_{i}}, \quad|p|=\mu-1 .
$$

It is easy to check that $\delta^{2}=0$.

Let $\Lambda_{\mu}^{\mu+1, r}$ be the kernel of $\delta: \Sigma_{\mu}^{\mu+1, r} \rightarrow \Sigma_{\mu-1}^{\mu, r+1}$. One has the following sequence:

$$
0 \longrightarrow \Lambda_{\mu}^{\mu+1, r} \longrightarrow \Sigma_{\mu}^{\mu+1, r} \stackrel{\delta}{\longrightarrow} \Lambda_{\mu-1}^{\mu, r+1} \longrightarrow 0
$$

since $\delta^{2}=0$. But this sequence is not exact, in general. However ( $\delta$-Poincaré lemma), it has been proved ([1], [4(a)], [5]) that there exists a positive number $\mu_{1} \geqq \mu_{0}, \mu_{1}$ depending only on $\mu_{0}, n, m$, and $l$, such that the sequence is exact for $\mu \geqq \mu_{1}$. We can choose a norm in $\Sigma_{\mu}^{\mu+1, r}$ by defining, for a form $\sigma=\sigma_{p, j_{1} \cdots j_{r}}^{j} d x^{j_{1}} \wedge \cdots \wedge d x^{j_{r}}$, belonging to $\Sigma_{\mu}^{\mu+1, r},\|\sigma\|_{\mu}=\sup \left|\sigma_{p, j_{1} \cdots j_{r}}^{j}(x)\right|$ for $x \in U$, all $j, j_{1}, \cdots, j_{r}$ and all the $p$ of length $\mu$. It has been proved (see [5]) that, choosing the coordinate $\left(x^{1}, \cdots, x^{n}\right)$ appropriately, one can assert the existence of a splitting of the sequence by a map

$$
\rho_{\mu}: \Lambda_{\mu-1}^{\mu, r+1} \longrightarrow \Sigma_{\mu}^{\mu+1, r}
$$

such that $\left\|\rho_{\mu}\right\|<C$ where the constant $C$ depends only on $\mathscr{D}$ and the choice of the coordinate. In particular, $C$ does not depend on $\mu$. One can, in the same way, define the map $\delta$ on $J_{\mu-1}^{\mu}(E)$, kernel of the map

$$
\pi^{\mu}: J^{\mu}(E) \longrightarrow J^{\mu-1}(E) \text {. }
$$

Since $J^{\mu}$ is locally isomorphic to $\bigoplus_{\nu=0}^{r} J_{\nu-1}^{\nu}, \delta$ can be regarded as a map

$$
\delta: J^{\mu, r} \longrightarrow J^{\mu-1, r+1} .
$$

But an element of $\Sigma^{\mu, r}$ is not in general sent into $\Sigma^{\mu-1, r+1}$, under $\delta$. (It is locally true, however, when the coefficients of $\mathscr{D}$, the matrices $a^{p}$, are constant.) 
Again, except in the constant coefficient case, the operator $d$, exterior derivation on the components of a jet, which are differential forms, does not send $\Sigma^{\mu, r}$ into $\Sigma^{\mu, r+1}$. But, roughly speaking, the difference $d-\delta$, between "actual" and "formal" derivations, preserves the structure. More precisely, there is a map

$$
D^{\mu}: \Sigma^{\mu, r} \longrightarrow \Sigma^{\mu-1, r+1},
$$

which is locally defined by

$$
D^{\mu} \sigma^{\mu}=d\left(\pi^{\mu} \sigma^{\mu}\right)-\delta \sigma^{\mu} .
$$

We shall often abbreviate $D^{\mu}$ by $D$, incorporating also in the same notation the projective limit $D^{\infty}$ of $D^{\mu}$, which sends $\Sigma^{\infty, r}$ into $\Sigma^{\infty, r+1}$. (For a more intrinsic definition of $D$, see [3].)

We come now to the resolution of the sheaf $\Theta$ of germs of solutions of the differential equation $\mathscr{D} f=0$. This resolution is the sequence (cf. $4(\mathrm{c}))$

$$
0 \longrightarrow \Theta \stackrel{\iota}{\longrightarrow} \Sigma^{\infty, 0} \stackrel{D}{\longrightarrow} \Sigma^{\infty, i} \longrightarrow \cdots \stackrel{D}{\longrightarrow} \Sigma^{\infty, n} \longrightarrow 0
$$

where

$$
(e(f))_{p}=\frac{\partial^{|p|} f}{\partial x^{p}}
$$

It is easily checked that $D \circ \iota=0$ and that the sequence is exact at $\Sigma^{\infty, 0}$. But it is not usually exact any further. When it is exact, we say that the $D$ (or better $D^{\infty}$ )-Poincare lemma is true.

Note that the $D^{\infty}$-Poincare lemma is equivalent to the $D^{\mu}$-Poincaré lemma for $\mu \geqq \mu_{1}$. We shall here indicate the details of the proof, for we shall use the process later, in the evaluation of the estimates.

First let us suppose that the $D$-Poincare lemma is true for some $\mu \geqq \mu_{1}$; and let us consider a $\sigma^{\infty}$ belonging to $\Sigma^{\infty, r}$ and satisfying $D \sigma^{\infty}=0$. The projection $\sigma^{\mu}$ of $\sigma^{\infty}$ in $\Sigma^{\mu, r}$ satisfies $D^{\mu} \sigma^{\mu}=0$, and thus there exists a $\tau^{\mu+1} \in \Sigma^{\mu+1, r-1}$ such that $D^{\mu+1} \tau^{\mu+1}=\sigma^{\mu}$. Let $\tau^{\infty}$ be any prolongation of $\tau^{\mu+1}$ and let $\tilde{\sigma}^{\infty}$ be $D \tau^{\infty}$. The form $\sigma^{\infty}-\tilde{\sigma}^{\infty}$ is closed under $D$ and belongs to the kernel, $\Sigma_{\mu, r}$, of the projection $\Sigma^{\infty, r} \rightarrow \Sigma^{\mu, r}$. Now the $D$-Poincaré lemma is trivially true for $\Sigma_{\mu}$ : no integration is necessary-it suffices to apply infinitely many times the $\delta$-Poincaré lemma. Therefore, $\sigma^{\infty}-\widetilde{\sigma}^{\infty}$ is a $D$-coboundary and so is $\sigma^{\infty}$.

Conversely, suppose that the $D^{\infty}$-Poincaré lemma is true, and let $\sigma^{\mu}$ be an element of $\Sigma^{\mu, r}$ such that $D^{\mu} \sigma^{\mu}=0, \mu \geqq \mu_{1}$. Since $\Sigma^{\mu+1} \rightarrow \Sigma^{\mu}$ is surjective, there exists a prolongation of $\sigma^{\mu}$ in $\Sigma^{\mu+1}$. Let $\widetilde{\sigma}^{\mu+1}$ be any such prolongation. Then $D^{\mu+1} \widetilde{\sigma}^{\mu+1}$ is actually an element of $\sum_{\mu-1}^{\mu, r+1}$, closed under $\delta$, therefore it belongs to $\Lambda_{\mu-1}^{\mu, r+1}$. We can now apply the 
$\delta$-Poincaré lemma, asserting the existence of a $\tau_{\mu}^{\mu+1} \in \Sigma_{\mu}^{\mu+1, r}$ such that $\delta \tau_{\mu}^{\mu+1}=D^{\mu+1} \widetilde{\sigma}^{\mu+1}$. By construction, $\sigma^{\mu+1}=\tilde{\sigma}^{\mu+1}+\tau_{\mu}^{\mu+1}$ belongs to $\Sigma^{\mu+1, r}$, projects onto $\sigma^{\mu}$, and is closed under $D^{\mu+1}$. This process can be repeated, thus giving us an infinite prolongation, $\sigma^{\infty}$, of $\sigma^{\mu}$, satisfying $D \sigma^{\infty}=0$. Therefore there exists a $\tau^{\infty}$ such that $D \tau^{\infty}=\sigma^{\infty}$, and it suffices now to project $\tau^{\infty}$ into $\Sigma^{\mu+1, r-1}$, in order to obtain a $\tau^{\mu+1}$ such that $D^{\mu+1} \tau^{\mu+1}=\sigma^{\mu}$.

It has been proved (see [3]) that the resolution is exact at $\Sigma^{\infty, 1}$ if and only if the nonhomogeneous equation $\mathscr{D} f=g$, where $g$ satisfies the required compatibility conditions, is locally solvable. But this equation is not always locally solvable, as Quillen has pointed out, namely the equation (H. Lewy's example)

$$
\frac{\partial f}{\partial \bar{z}}-i z \frac{\partial f}{\partial x^{3}}=g
$$

where $z=x^{1}+i x^{2}, \bar{z}=x^{1}-i x^{2}$, has a solution if $g$ is analytic, but no solution in general if $g$ is $C^{\infty}$. Therefore, the $D$-Poincare lemma is generally false. However, we can say that

(i) It is "formally" true (see [3]).

(ii) It is true in the constant coefficient case, in view of theorems of Ehrenpreis and Malgrange.

(iii) It is true in the analytic case. Analytic means here that the coefficients of the equation $\mathscr{D} f=0$, and the jet $\sigma$ are analytic (cf. Lewy's equation).

The exactness in case (iii) is known (see [3], [4]). However, our purpose in this paper is to give a new simplified proof of the exactness in case (iii) by defining a natural homotopy operator $K$, which, we think, is natural in the analytic category, but, (see end of $\S 7$ ) may be defined under weaker assumptions of analyticity, i.e. supposing only that the coefficients of $\mathscr{D}$ are analytic and the Spencer sequence is exact.

3. Definition of the operator $K$. Let us first recall the existence of an operator $k$, acting on ordinary differential forms defined in an open set $U$, starlike with respect to the origin, in which coordinates $\left(x^{i}\right)$ have been chosen, and satisfying:

$$
\left\{\begin{aligned}
k d \sigma+d k \sigma & =\sigma & & \text { if the degree of } \sigma \text { is positive } \\
& =\sigma(x)-\sigma(0) & & \text { if the degree of } \sigma \text { is } 0 .
\end{aligned}\right.
$$

The operator $k$ may be obtained in the following way. One uses the interior product $\sigma \lambda x$ of the differential form $\sigma=\sigma_{j_{1} \cdots j_{r}} d x^{j_{1}} \wedge \cdots \wedge d x^{j_{r}}$ by the vector field $x=\sum_{1}^{n} x^{k}\left(\partial / \partial x^{k}\right)$, which is defined by 


$$
\sigma \pi x=\sigma_{j_{1} \cdots j_{r}}\left(\sum_{k=1}^{r}(-1)^{k-1} x^{j_{k}} d x^{j_{1}} \wedge \cdots \wedge \widehat{d x^{j_{k}}} \wedge \cdots \wedge d x^{j_{r}}\right)
$$

in positive degree, $\sigma \pi x=0$ in degree 0 . Let us recall that the operator $\pi x$ is a derivation:

$$
(\alpha \wedge \beta) \pi x=(\alpha \pi x) \wedge \beta+(-1)^{r} \alpha \wedge(\beta \pi x)
$$

where $r$ is the degree of $\alpha$. Then one defines the operator $V \sigma$ (Lie derivative) by

$$
V \sigma=d \sigma \pi x+d(\sigma \pi x)
$$

An easy computation shows that

$$
V \sigma=\left(\sum_{j_{0}=1}^{n} x^{j_{0}} \frac{\partial \sigma_{j_{1} \cdots j_{r}}}{\partial x^{j_{0}}}+r \sigma_{j_{1} \cdots j_{r}}\right) d x^{j_{1}} \wedge \cdots \wedge d x^{j_{r}},
$$

thus proving that $V$ is invertible on the differential forms of positive degree. In fact, the equation

$$
\sum_{i=1}^{n} x^{i} \frac{\partial f}{\partial x^{i}}+r f=g
$$

has a unique $C^{\infty}$ solution, when $g$ is $C^{\infty}$, given by

$$
f=\int_{0}^{1} t^{r-1} g\left(t x^{1}, \cdots, t x^{n}\right) d t \quad r \geqq 1 .
$$

Let $k \sigma$ be

$$
\begin{cases}V^{-1} \sigma \pi x & \text { in positive degree } \\ 0 & \text { in degree } 0\end{cases}
$$

It is now easy to check that $k$ satisfies (1), and therefore, as is well known, $k$ can be used to prove that a differential form closed under $d$ is locally a $d$-coboundary ( $d$-Poincaré lemma).

We would like to generalize this process, in order to obtain an operator $K$, acting on the differential forms $\sigma \in \Sigma^{\infty, r}$, and satisfying

$$
D K \sigma+K D \sigma=\sigma
$$

in positive degree. We recall that we are here working in a neighborhood $U$ of a point 0 of the manifold, covered by a coordinate $\left(x^{i}\right)$.

We can apply $k$ to all the components of $\sigma$, and define the operator $W$ by

$$
W \sigma=D k \sigma+k D \sigma .
$$

But here the operator $W$ is not generally invertible, and it is not so easy to see directly when it is invertible. We have 


$$
\begin{aligned}
D k \sigma+k D \sigma & =(d k+k d)(\sigma)-(\delta k+k \delta)(\sigma) \\
& = \begin{cases}\sigma-(\delta k+k \delta)(\sigma) & \text { in positive degree } \\
\sigma(x)-\sigma(0)-k \delta \sigma & \text { in degree } 0 .\end{cases}
\end{aligned}
$$

Symbolically speaking, $W$ will be invertible if the series $\sum_{l=0}^{\infty}(\delta k+k \delta)^{l}$ converges, or, equivalently, if the series $\sum_{l=0}^{\infty}(\delta k)^{l}$ does. We shall define

$$
K \sigma=
$$

$$
\begin{cases}k \sum_{l=0}^{\infty}(\delta k)^{l} \sigma & \text { in positive degree, when the series is convergent, } \\ 0 & \text { in degree } 0 .\end{cases}
$$

The operator $K$ has been constructed in order to verify (2), but it is necessary to check it, because of the slight irregularities introduced by the forms of degree 0 . If both $\sum_{l=0}^{\infty}(\delta k)^{l} \sigma$ and $\sum_{l=0}^{\infty}(\delta k)^{l} D \sigma$ converge,

$$
\begin{aligned}
D K \sigma+K D \sigma & =d k \sum_{l=0}^{\infty}(\delta k)^{l} \sigma-\sum_{l=1}^{\infty}(\delta k)^{l} \sigma+k \sum_{l=0}^{\infty}(\delta k)^{l} D \sigma \\
& =\sum_{l=0}^{\infty}(\delta k)^{l} \sigma-k d \sum_{l=0}^{\infty}(\delta k)^{l} \sigma-\sum_{l=1}^{\infty}(\delta k)^{l} \sigma+k \sum_{l=0}^{\infty}(\delta k)^{l} D \sigma \\
& =\sigma+k \sum_{l=0}^{\infty}\left((\delta k)^{l} D \sigma-d(\delta k)^{l} \sigma\right)
\end{aligned}
$$

and the last sum is easily seen to be equal to zero, by using $d k+$ $k d=$ identity, and $d \delta+\delta d=0$.

In degree $0,(2)$ is no longer true, but we find,

$$
K D \sigma=\sigma(x)-\sum_{l=0}^{\infty}(k \delta)^{l} \sigma(0)
$$

if the last series is convergent. The component

$$
\left(\sum_{l=0}^{\infty}(k \delta)^{l} \sigma(0)\right)_{p}
$$

is a power series in $x^{1}, \cdots, x^{n}$, actually the Taylor expansion of an analytic function $\varphi_{p}$, whose derivatives at the origin are

$$
\frac{\partial^{|q|} \varphi_{p}}{\partial x^{q}}(0)=\sigma_{p+q}(0) \text {. }
$$

In other words, there exists a function $\varphi$, analytic in $U$, such that $\partial^{|p|} \varphi / \partial x^{p}=\varphi_{p}$, thus showing that $\sum_{l=0}^{\infty}(k \delta)^{l} \sigma(0)=\iota^{\infty}(\varphi)$. It can be seen that the function $\varphi$ belongs, in fact, to $\Theta$. The demonstration is analogous to the one we shall give later in order to prove that $K$ preserves $\Sigma^{\infty, *}$. 
Everything we have said in this paragraph applies to the "formal" case, in the sense that it is correct for formal power series.

REMARK. Among formal homotopy operators on $\Sigma^{\infty, *}$, the above homotopy operator is uniquely determined by the following conditions:

(i) $D K+K D=I$ in positive degree;

(ii) $K \sigma$ is in the image of the derivation $\pi x$, that is $K \sigma$ has the form $K \sigma=\omega \pi x$.

We have defined such an operator, symbolically written

$$
K=k \sum_{0}^{\infty}(\delta k)^{l} \text {. }
$$

Therefore, this particular operator has the additional property that

(iii) $K(\omega \pi x)=0$.

As a consequence, we observe that $K^{2}=0$.

Now let $K^{\prime}$ be any other (formal) operator satisfying (i) and (ii). Then, in positive degree, we have

$$
D\left(K-K^{\prime}\right)+\left(K-K^{\prime}\right) D=0 .
$$

Applying on the left the operator $K$, we obtain, by using (ii) and (iii),

$$
K D\left(K-K^{\prime}\right)=0 .
$$

But $K D=I-D K$ in positive degree, and also in degree 0 when applied to a jet form vanishing at 0 . Therefore,

$$
K-K^{\prime}=0 \text {. }
$$

4. Explicit expression for $K$, applied to a form of positive degree. It is enough, because $K$ is linear, to make the computation when all the components of $\sigma$ involve the same differential element $d x^{J}=d x^{j_{1}} \wedge \cdots \wedge d x^{j_{r}}$,

We shall compute the first two terms of the series, and the generalization is straightforward.

Let $\sigma$ be $\left(\sigma_{p} d x^{J}\right)$. Then

$$
\begin{aligned}
(k \sigma)_{p} & =\left(\int_{0}^{1} t^{r-1} \sigma_{p}(t x) d t\right)\left(d x^{J} \pi x\right) \\
(\delta k \sigma)_{p} & =\sum_{i=1}^{n}\left(\int_{0}^{1} t^{r-1} \sigma_{p+1_{i}}(t x) d t\right) d x^{i} \wedge\left(d x^{J} \pi x\right), \\
(k \delta k \sigma)_{p} & =\sum_{i=1}^{n}\left(\int_{0}^{1} u^{r}\left(\int_{0}^{1} t^{r-1} \sigma_{p+1_{i}}(t u x) d t\right) d u\right)\left(d x^{i} \wedge\left(d x^{J} \pi x\right)\right) \pi x \\
& =\sum_{i=1}^{n} x^{i}\left(\int_{0}^{1}\left(\int_{0}^{u} t^{r-1} \sigma_{p+1_{i}}(t x) d t\right) d u\right)\left(d x^{J} \pi x\right) \\
& =\sum_{i=1}^{n} x^{i}\left(\int_{0}^{1}(1-t) t^{r-1} \sigma_{p+1_{i}}(t x) d t\right)\left(d x^{J} \pi x\right) .
\end{aligned}
$$


More generally,

$$
\left(k(\delta k)^{l} \sigma\right)_{p}=\tau_{p}^{l} d x^{J} \pi x
$$

where

$$
\tau_{p}^{l}=\int_{0}^{1} t^{r-1}(1-t)^{l}\left(\sum_{|q|=l} \frac{x^{q}}{q !} \sigma_{p+q}(t x)\right) d t
$$

and

$$
K \sigma=\left(\tau_{p} d x^{J} \pi x\right)
$$

where

$$
\tau_{p}=\sum_{l=0}^{\infty} \tau_{p}^{l}
$$

if the series converges.

5. Sufficient condition for the existence of $K \sigma$. Let $V$ be a compact set contained in $U$, and let $\sigma$ be an element of $\Sigma^{\infty, r}$, with components

$$
\sigma_{p, j_{1}, \ldots, j_{r}}^{j} d x^{j_{1}} \wedge \cdots \wedge d x^{j_{r}} \quad j=1,2, \cdots, m .
$$

We recall that $\|\sigma\|_{\mu}=\sup \left|\sigma_{p, j_{1}, \ldots, j_{r}}^{j}(x)\right|$ for $x \in V$, all $j, j_{1}, \cdots, j_{r}$, and all the $p$ of length $\mu$. We shall say that $\sigma$ satisfies the condition (C) if and only if there exist two numbers $M$ and $h$, finite and positive, such that

$$
\|\sigma\|_{\mu} \leqq M \mu ! h^{\mu}
$$

for all $\mu$.

Condition (C) means that, as far as their growth is concerned, the $\sigma_{p}$ behave like the derivatives of an analytic $\boldsymbol{R}^{m}$-valued function.

With the notations of $\S 4$, we have, when (C) is fulfilled,

$$
\left|\tau_{p}^{l}\right| \leqq M(|p|+l) ! h^{|p|+l} \frac{(\rho(x))^{l}}{l !}
$$

where $\rho(x)=\sum_{i=1}^{n}\left|x^{i}\right|$. We can suppose that $U$ is small enough to insure

$$
\rho(x)<\frac{1}{h}
$$

and the series $\tau_{p}$ is now uniformly convergent (for $\rho(x) \leqq(1 / h)-\varepsilon$ ), for all $p$. Therefore, in this case, $K \sigma$ converges, and we can permute integration and summation, thus obtaining 


$$
K \sigma=\left(\int_{0}^{1} t^{r-1}\left(\sum_{i=0}^{\infty}(1-t)^{l}\left(\sum_{|q|=l} \frac{x^{q}}{q !} \sigma_{p+q}(t x)\right)\right) d t\right) d x^{J} \pi x .
$$

6. Conservation by $K$ of the structure of $\Sigma^{\infty, *}$. In $\S 4$, we have associated, to the form $\sigma=\left(\sigma_{p}\right) d x^{J}$, the form $K \sigma=\left(\tau_{p}\right) d x^{J} \pi x$, at least formally. We want to know whether $\tau=\left(\tau_{p}\right)$ satisfies the equations $\mathscr{L}_{q}(\tau)=0$ (see $\S 1$ ) as $\tilde{\sigma}=\left(\sigma_{p}\right)$ does. Our proof will show that this is formally true, but in order to prove that it is actually true, we shall need some assumptions of analyticity.

LemMa 1. If there exists a number $A$, such that, for $\rho(x)<A$, the $\sigma_{p}(x)$ are analytic, for all $p$, and satisfy the condition (C), then $K \sigma$ exists locally and belongs to $\Sigma^{\infty}, *$.

Lemma 2. If there exists a number $A$, such that, for $\rho(x)<A$, the coefficients of $\mathscr{D}$ are analytic, and $\sigma$ satisfies the condition (C), then $K \sigma$ exists locally and belongs to $\Sigma^{\infty}, *$.

Proof of Lemma 1. The equation $\mathscr{L}_{q}(x)(\sigma(x))=0$ actually involves only the $\sigma_{p}$ with $|p| \leqq|q|+\mu_{0}$. In $\mathscr{L}_{q}(x)(\sigma)$ let us replace each $\sigma_{p}(x)$ by

$$
\widetilde{\tau}_{p}(t, x)=\sum_{l=0}^{\infty}(1-t)^{l} \sum_{|s|=l} \frac{x^{s}}{s !} \sigma_{p+s}(t x)
$$

and let us call $\varphi_{q}(t, x)$ (or symbolically $\mathscr{L}_{q}(x)(\widetilde{\tau}(t, x))$ ) the result of this substitution. We shall prove that, for $x$ fixed and small enough, $\varphi_{q}(t, x)$ is identically zero, in a neighborhood of the closed interval $[0,1]$, and the desired result will follow directly by integration with respect to $t$.

Let us first remark that $\varphi_{q}(1, x)$ vanishes, because $\widetilde{\tau}_{p}(1, x)=\sigma_{p}(x)$ and therefore it satisfies the equation

$$
\mathscr{L}_{q}(x)(\widetilde{\tau}(1, x))=0 .
$$

Next, for $x$ fixed, $\rho(x)<A$, we see, by using (C), that the $\widetilde{\tau}_{p}(t, x)$ are analytic functions of $t$, in the domain

$$
|t|<\frac{A}{\rho(x)}, \quad|1-t|<\frac{1}{h \rho(x)} .
$$

If we impose, for example, the condition $\rho(x)<\inf (A / 2,1 / 2 h)$, the element $\widetilde{\tau}_{p}(t, x)$, and also $\varphi_{q}(t, x)$, will be analytic functions of $t$ for $-1<t<2$, i.e. in a neighborhood of [0.1]. It now remains to prove that all the derivatives of $\varphi_{q}(t, x)$ are zero for $t=1$. We remark, by using the analyticity of $\sigma_{p}$ and the condition (C), that all the series 
intervening in the argument can be differentiated term-by-term. For instance:

$$
\begin{aligned}
\frac{\partial \tilde{\tau}_{p}(t, x)}{\partial t}= & \sum_{i=0}^{\infty}(1-t)^{l}\left[\sum_{|s|=l} \sum_{i=1}^{n} \frac{x^{s+1}}{s !} \frac{\partial \sigma_{p+s}(t x)}{\partial x^{i}}-(l+1) \sum_{|s|=l+1} \frac{x^{s}}{s !} \sigma_{p+s}(t x)\right] \\
= & \sum_{i=0}^{\infty}(1-t)^{l} \sum_{|w|=l+1} x^{w}\left[\left(\sum_{i=1}^{n} \frac{1}{\left(w-1_{i}\right) !} \frac{\partial \sigma_{p+w-1_{i}}(t x)}{\partial x^{i}}\right)\right. \\
= & \left.-(l+1) \frac{\sigma_{q+w}(t x)}{w !}\right] \\
= & \sum_{i=0}^{\infty}(1-t)^{l} \sum_{|w|=l+1} \sum_{i=1}^{n} \frac{x^{w}}{\left(w-1_{i}\right) !}\left[\frac{\partial \sigma_{p+w-1_{i}}}{\partial x^{i}}(t x)-\sigma_{p+w}(t x)\right] \\
= & \sum_{i=1}^{n} x^{i} \sum_{i=0}^{\infty}(1-t)^{l} \sum_{|s|=l} \frac{x^{s}}{s !}\left(\frac{\partial \sigma_{p+s}}{\partial x^{i}}(t x)-\sigma_{p+s+1}(t x)\right) .
\end{aligned}
$$

We have denoted by $\widetilde{\sigma}$ the elements of $\Sigma^{\infty, 0}$ whose components are $\left(\sigma_{p}\right)_{\circ}$. Let us denote by $\alpha_{p, i} d x^{i}$ the components of $D \widetilde{\sigma}$, in other words

$$
\alpha_{p, i}=\frac{\partial \sigma_{p}}{\partial x^{i}}-\sigma_{p+1_{i}} .
$$

The derivative $\partial \widetilde{\tau}_{p}(t, x) / \partial t$ can now be written

$$
\frac{\partial \widetilde{\tau}_{p}}{\partial t}(t, x)=\sum_{i=1}^{n} x^{i} \widetilde{\tau}_{p, i},
$$

where

$$
\tilde{\tau}_{p, i}=\sum_{l=0}^{\infty}(1-t)^{l} \sum_{|s|=l} \frac{x^{s}}{s !} \alpha_{p+s, i}(t x) .
$$

We note that $\widetilde{\tau}_{p, i}$ has the same form as $\widetilde{\tau}_{p}$. The element $\left(\sigma_{p}\right)$ has simply been replaced by $\left(\alpha_{p, i}\right)$, both belonging to $\Sigma^{\infty}$. In particular, $\widetilde{\tau}_{p, i}(1, x)=\alpha_{p, i}(x)$ and $\partial \varphi_{q}(t, x) / \partial t$ is zero for $t=1$. This process can obviously be repeated indefinitely, thus proving our assertion.

Proof of Lemma 2. Here we prove again that the function $\varphi_{q}(t, x)$ is identically zero in a neighborhood of $[0,1]$ for $x$ small enough, but in order to do it we shall make the change of variables

$$
t x=X, \quad \frac{1}{t}=T .
$$

The function $\varphi_{q}(t, x)$ becomes

$$
\hat{\varphi}_{q}(T, X)=\mathscr{L}_{q}(T X)(\hat{\tau}(T, X)),
$$

where this expression involves the terms $\hat{\tau}_{p}(T, X)$ for $|p| \leqq|q|+\mu_{0}$. and 


$$
\hat{\tau}_{p}(T, X)=\sum_{l=0}^{\infty}(T-1)^{l} \sum_{|s|=l} \frac{X^{s}}{s !} \sigma_{p+s}(X) .
$$

As in the preceding proof, we see that $\hat{\varphi}_{q}(1, X)=0$. Since $\mathscr{L}_{q}(T X)$ is analytic, and $\hat{\tau}_{q}(T, X)$ is a power series in $(T-1)$, we see that $\hat{\varphi}_{q}(T, X)$ is, for $X$ fixed, $\rho(X)<A$, an analytic function of $T$ in the domain $|T| \rho(X)<A,|T-1| \rho(X)<1 / h$, which is simply the transform of the domain previously considered, i.e. the domain

$$
\rho(x)<A, \quad|1-t| \rho(x)<1 / h,
$$

with the condition $|t| \rho(x)<A$.

Therefore, if we can show that all the derivatives of $\hat{\varphi}_{p}(T, X)$ vanish for $T=1$, our proof will be complete.

Here the series intervening in $\varphi_{q}$ are power series in $(T-1)$, therefore they can be differentiated term-by-term, and we have, symbolically:

$$
\frac{\partial \hat{\varphi}_{q}(T, X)}{\partial T}=\sum_{i=1}^{n} X^{i} \frac{\partial \mathscr{L}_{q}(T X)}{\partial x^{i}}(\hat{\tau}(T, X))+\mathscr{L}_{q}(T X)\left(\frac{\partial \hat{\tau}}{\partial T}(T, X)\right) .
$$

But

$$
\begin{aligned}
\frac{\partial \hat{\tau}_{p}}{\partial T}(T, X) & =\sum_{l=0}^{\infty}(l+1)(T-1)^{l} \sum_{\mid s i=l+1} \frac{X^{s}}{s !} \sigma_{p+s}(X) \\
& =\sum_{i=1}^{n} X^{i} \sum_{i=0}^{\infty}(l+1)(T-1)^{l} \sum_{|s|=l+1} \frac{X^{s-1} i}{s !} \sigma_{p+s}(X) .
\end{aligned}
$$

An easy computation now shows that

$$
\frac{\partial \widehat{\varphi}_{q}(T, X)}{\partial T}=\sum_{i=1}^{n} X^{i} \mathscr{L}_{q++_{i}}(T X)(\hat{\tau}(T, X)) .
$$

Again all the derivatives of $\hat{\varphi}_{q}(T, X)$ will have similar forms, and will vanish for $T=1$.

These two lemmas prove that, whenever $K \sigma$ converges, it respects the structure of $\Sigma^{\infty}, *$, if either the coefficients of $\mathscr{D}$, or the components of $\sigma$ are analytic. Unfortunately, as we shall see in the next paragraph, we need the analyticity of both coefficients and components to obtain an element satisfying the condition (C).

However, in the constant coefficient case, and more generally under the assumption that the resolution associated with some operator $\mathscr{D}$, with analytic coefficients, is exact, it will be possible to prove that, given a $\sigma^{\mu} \in \Sigma^{\mu, r}$, closed under $D^{\mu}$, $\sigma^{\mu}$ can be prolonged to a $\sigma^{\infty} \in \Sigma^{\infty, r}$, closed under $D$, in such a way that $(\mathrm{C})$ is fulfilled by $\sigma^{\infty}$, i.e. $K \sigma^{\infty}$ exists.

In order to prove that the resolution is exact, in the case where 
both the coefficients of $\mathscr{D}$ and components of $\sigma^{\mu} \in \Sigma^{\mu, r}$ are analytic, we shall show that a $\sigma^{\mu}$ closed under $D^{\mu}$, can be prolonged to a $\sigma^{\infty}$ closed under $D$, in such a way that $(\mathrm{C})$ is fulfilled by $\sigma^{\infty}$, i.e. $K \sigma^{\infty}$ exists.

\section{Estimates.}

Lemma 3. If the coefficients of $\mathscr{D}$ are analytic, any $\sigma^{\mu} \in \Sigma^{\mu, r}$ can be prolonged to a $\sigma^{\infty} \in \Sigma^{\infty, r}$ in such a way that $\sigma^{\infty}$ satisfies the condition (C).

Proof. It is obviously sufficient to consider only the forms of degree 0 .

Let

$$
\mathscr{D} f=\sum_{i=1}^{n} A_{i} \frac{\partial f}{\partial x^{i}}+B f,
$$

where the $A_{i}$ and $B$ are $l \times m$ matrix-valued functions. (One can always reduce to this case. Cf. Quillen's thesis.) Here we can write explicitly our equations $\mathscr{L}_{p}(\sigma)=0$, in the form

$$
\left\{\begin{array}{l}
\mathscr{L}_{0}(x)(\sigma)=\sum_{i} A_{i} \sigma_{0+1_{i}}+B \sigma_{0} \\
\mathscr{L}_{p}(x)(\sigma)=\sum_{i, q \subset p} r_{q} \frac{\partial A_{i}}{\partial x^{q}} \sigma_{p-q+1_{i}}+\sum_{q \subset p} r_{q} \frac{\partial B}{\partial x^{q}} \sigma_{p-q}
\end{array}\right.
$$

where the integers $r_{q}$ are multinomial coefficients, coming from Leibnitz' formula.

We know that the map $\Sigma^{\mu+1} \rightarrow \Sigma^{\mu}$ is surjective; therefore, there exists an element $\bar{\sigma}_{\mu}^{\mu+1}$, belonging to $J_{\mu}^{\mu+1}(E)$, such that $\sigma^{\mu}+\bar{\sigma}_{\mu}^{\mu+1}$ belongs to $\Sigma^{\mu+1}$. In other words, if we denote by $\bar{\sigma}^{\mu+1}$ the sum $\sigma^{\mu}+\bar{\sigma}_{\mu}^{\mu+1}$, we have

$$
\mathscr{L}_{p}\left(\bar{\sigma}^{\prime \prime+1}\right)=0
$$

for all $p$ satisfying $|p| \leqq \mu$. In particular,

$$
-\rho\left(\bar{\sigma}_{\mu}^{\mu+1}\right)=\sum_{i=1}^{n} \sum_{\substack{q \subset p \\|q| \geqslant 1}} r_{q} \frac{\partial A_{i}}{\partial x^{q}} \sigma_{p-q+1_{i}}+\sum_{q \subset p} r_{q} \frac{\partial B}{\partial x^{q}} \sigma_{p-q},
$$

where $\rho$ denotes the symbol of $\mathscr{D}$. (See [3] for the definition of the symbol.)

It has been proved in [5] that there exists a map $r$, defined on $\rho\left(J_{\mu}^{\mu+1}(E)\right)$ restricted to a neighborhood $U$ of the origin, such that $\rho \circ r=$ identity, and $\|r\| \leqq H$, uniformly in this neighborhood, where $H$ is a constant independent of $\mu$. 
This proposition enables us to replace $\bar{\sigma}_{\mu}^{\mu+1}$ by a $\sigma_{\mu}^{\mu+1}$ satisfying $\rho\left(\sigma_{\mu}^{\mu+1}\right)=\rho\left(\bar{\sigma}_{\mu}^{\mu+1}\right)$ and $\left\|\sigma_{\mu}^{\mu+1}\right\| \leqq H\left\|\rho\left(\bar{\sigma}_{\mu}^{\mu+1}\right)\right\|$. In other words, $\sigma_{\mu}^{\mu+1}$ will be bounded in terms of the derivatives of $A_{i}$ and $B$, and also of the components $\sigma_{\nu-1}^{\nu}$ of $\sigma^{\mu}$ in $J_{\nu-1}^{\nu}, 0 \leqq \nu \leqq \mu$. We call $M_{\nu}$ the bound of $\sigma_{\nu-1}^{\nu}$.

Now, since the functions $A_{i}$ and $B$ are analytic, we can assert the existence of two numbers $N$ and $H_{1}$, and of a compact set $V$ contained in $U$, such that, in $V$

$$
\left\|\frac{\partial B}{\partial x^{q}}\right\| \leqq N H_{1}^{|q|}|q| !
$$

and

$$
\left\|n \frac{\partial A_{i}}{\partial x^{q}}\right\| \leqq N H_{1}^{|q|-1}(|q|-1) ! .
$$

In the last inequality, we are actually using the analyticity of the first derivatives of $A_{i}$, and the purpose is simply to make the computation easier. We shall call $h$ the supremum of $H$ and $H_{1}$.

Now we have, in $V$

$$
\left\|\sum_{q \subset p} r_{q} \frac{\partial B}{\partial x^{q}} \sigma_{p-q}\right\| \leqq N \sum_{\nu=0}^{\mu} C_{\mu}^{\nu} h^{\nu} \nu^{\prime} \cdot M_{\mu-\nu}
$$

where

$$
C_{\mu}^{\nu}=\frac{\mu !}{\nu !(\mu-\nu) !}
$$

In the same manner

$$
\left\|\sum_{i=1}^{n} \sum_{\substack{q \subset p \\|q| \geqq 1}} r_{q} \frac{\partial A_{i}}{\partial x^{q}} \sigma_{p-q+1_{i}}\right\| \leqq N \sum_{\nu=1}^{\mu} C_{\mu}^{\nu} h^{\nu-1}(\nu-1) ! M_{\mu-\nu+1}
$$

i.e.,

$$
\left\|\sum_{i=1}^{n} \sum_{\substack{q \subset p \\|q| \geqq 1}} r_{q} \frac{\partial A_{i}}{\partial x^{q}} \sigma_{p-q+1}\right\| \leqq N \sum_{\nu=0}^{\mu-1} C_{\mu}^{\nu+1} h^{\nu} \nu ! M_{\mu_{-\nu}} .
$$

Finally, by using the equality $C_{\mu}^{\nu}+C_{\mu}^{\nu+1}=C_{\mu+1}^{\nu+1}$, we have

$$
\left\|\rho\left(\bar{\sigma}_{\mu}^{\mu+1}\right)\right\| \leqq N \sum_{\nu=0}^{\mu} C_{\mu+1}^{\nu+1} h^{\nu} \nu ! M_{\mu-\nu}
$$

and

$$
M_{\mu+1} \leqq N h \sum_{\nu=0}^{\mu} C_{\mu+1}^{\nu+1} h^{\nu} \nu ! M_{\mu-\nu} .
$$


We can always suppose that, in $V$

$$
M_{\nu} \leqq N M_{0} h^{\nu}(l+N)^{\nu} \nu !, \quad \text { for } 1 \leqq \nu \leqq \mu .
$$

It follows that

$$
\begin{aligned}
M_{\mu+1} & \leqq N M_{0} h^{\mu+1}\left(\mu !+(\mu+1) ! \sum_{\nu=0}^{\mu-1} \frac{N(1+N)^{\mu-\nu}}{\nu+1}\right) \\
& \leqq N M_{0} h^{\mu+1}(1+N)^{\mu+1}(\mu+1) !
\end{aligned}
$$

We call $\sigma^{\mu+1}$ the sum $\sigma_{\mu}^{\mu+1}+\sigma^{\mu}$, and define, in the same way, an element $\sigma_{\mu+1}^{\mu+2}$ satisfying $\sigma^{\mu+1}+\sigma_{\mu+1}^{\mu+2} \in \Sigma^{\mu+2}$ and

$$
M_{\mu+2}=\left\|\sigma_{\mu+2}^{\mu+2}\right\| \leqq N M_{0} h^{\mu+2}(1+N)^{\mu+2}(\mu+2) !
$$

and this can be repeated indefinitely, thus proving the existence of an infinite prolongation of $\sigma^{\mu}$, satisfying the condition (C).

REMARK. In this case, i.e., when the coefficients of $\mathscr{D}$ are analytic, the resolution is exact at $\Sigma^{\infty, n}$. In fact, this is equivalent to saying (see $\S 2$ ) that the map $D^{\mu+1}: \Sigma^{\mu+1, n-1} \rightarrow \Sigma^{\mu, n}$ is surjective for $\mu \geqq \mu_{1}$, and this map is actually surjective for all $\mu \geqq 1$. Indeed, as we have just seen, the element $\sigma^{\mu, n}$ can be prolonged to a $\sigma^{\infty, n}$, satisfying the condition (C), hence $K \sigma^{\infty, n}$ exists. But, in degree $n$, since $\sigma$ is automatically closed, $D K \sigma$ reduces to $\sigma$. Therefore, $\sigma^{\infty, n}$ is a $D$-coboundary, and $\sigma^{\mu, n}$ is consequently a $D^{\mu+1}$-coboundary.

This is, of course, exceptional; for the other degrees, in order to prove that the $D^{\mu}$-Poincaré lemma is true, we must be able to prolong a form $\sigma^{\mu, r}$ closed under $D^{\mu}$, to a form $\sigma^{\infty, r}$, closed under $D$ (this is possible, as we have seen in $\S 2$ ) and satisfying (C), and for the latter condition we shall need more assumptions, as will be seen in the following theorem.

Theorem. If the coefficients of $\mathscr{D}$ are analytic, and if $\sigma^{\mu, r}$ denotes an analytic jet form, closed under $D^{\prime \prime}$, where $\mu \geqq \mu_{1}, r>0$, $\sigma^{\mu, r}$ is locally a $D^{\mu+1}$-coboundary.

Proof. We shall prolong $\sigma^{\mu}$ into a form $\sigma^{\infty}$, closed under $D$ and satisfying (C). Let us first observe that the process used in the demonstration of Lemma 3 respects the analyticity; in fact, the map defined by Sweeney can be chosen to respect analyticity. Therefore, going back to the notations at the end of $\S 2$, we can choose an element $\widetilde{\sigma}_{\mu}^{\mu+1}$, analytic and satisfying

$$
\left\|\widetilde{\sigma}_{\mu}^{\mu+1}\right\| \leqq N h \sum_{\nu=0}^{\mu} C_{\mu+1}^{\nu+1} h^{\nu} \nu ! M_{\mu-\nu} .
$$


But, as we have seen in the proof of the fact that the $D^{\infty}$-Poincare lemma implies the $D^{\mu}$-Poincaré lemma, the desired element $\sigma_{\mu}^{\mu+1}$ (i.e. element satisfying $\left.D^{\mu+1}\left(\sigma^{\mu}+\sigma_{\mu}^{\mu+1}=0\right)\right)$ is obtained by adding to $\tilde{\sigma}_{\mu}^{\mu+1}$, an element $\tau_{\mu}^{\mu+1}$ such that

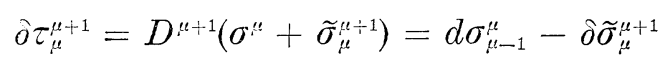

and we have (by the $\delta$-Poincaré lemma with bound)

$$
\left\|\tau_{\mu}^{\mu+1}\right\| \leqq C\left\|d \sigma_{\mu-1}^{\mu}-\delta \widetilde{\sigma}_{\mu}^{\mu+1}\right\| \text {. }
$$

The element $\sigma_{\mu-1}^{\mu}$ is analytic; therefore, in $V$,

$$
\left\|d \sigma_{i-1}^{\mu}\right\| \leqq n h\left\|\sigma_{\mu-1}^{\mu}\right\| \text {. }
$$

Furthermore,

$$
\left\|\delta \widetilde{\sigma}_{\mu}^{\mu+1}\right\| \leqq n\left\|\widetilde{\sigma}_{p}^{\mu+1}\right\| \text {. }
$$

By combining the last three inequalities, we find

$$
\left\|\sigma_{\mu}^{\mu+1}\right\| \leqq n C h M_{\mu}+(1+n C) N h \sum_{\nu=0}^{\mu} C_{\mu+1}^{\nu+1} h^{\nu} \nu ! M_{\mu-\nu} .
$$

Let $\lambda=N(1+n C)+n C$, and let us suppose, inductively, that

$$
M_{\nu} \leqq M_{0} \lambda^{\nu} h^{\nu} \nu ! \quad \text { for } 0 \leqq \nu \leqq \mu .
$$

Then

$$
\begin{aligned}
M_{\mu+1} \leqq & (1+n C) M_{0} N h^{\mu+1}(\mu+1) !\left(\lambda^{\mu}+\frac{\lambda^{\mu-1}}{2}+\cdots+\frac{\lambda}{\mu}+\frac{1}{\mu+1}\right) \\
& +n C M_{0} \lambda^{\prime \prime} h^{\mu+1} \mu ! .
\end{aligned}
$$

The last bound can be written

$$
\begin{aligned}
& M_{0} h^{\mu+1}(\mu+1) !\left(\lambda^{\mu+1}+\frac{\lambda^{\mu}}{2}+\cdots+\frac{\lambda^{2}}{\mu}+\frac{\lambda}{\mu+1}\right) \\
& \quad+n C M_{0} h^{\mu+1}(\mu+1) !\left[\frac{\lambda^{\mu}}{\mu+1}-\lambda^{\mu}-\frac{\lambda^{\mu-1}}{2}-\cdots-\frac{\lambda}{\mu}-\frac{1}{\mu+1}\right] \\
& =M_{0} h^{\mu+1}(\mu+1) ! \lambda^{\mu+1}+M_{0} h^{\mu+1}(\mu+1) ! \Delta
\end{aligned}
$$

where

$$
\begin{aligned}
\Delta=\left(\frac{\lambda^{\mu}}{2}+\right. & \left.\cdots+\frac{\lambda^{2}}{\mu}+\frac{\lambda}{\mu+1}\right) \\
& -n C\left(\frac{\mu}{\mu+1} \lambda^{\prime \prime}+\frac{\lambda^{\mu-1}}{2}+\cdots+\frac{\lambda}{\mu}+\frac{1}{\mu+1}\right) .
\end{aligned}
$$

It remains to show that $\Delta$ is negative. Since $n C$ can be taken larger than 1, it will be sufficient to prove that 


$$
\frac{\lambda^{\mu}}{2}+\cdots+\frac{\lambda^{2}}{\mu}+\frac{\lambda}{\mu+1}<\frac{\mu \lambda^{\mu}}{\mu+1}+\frac{\lambda^{\mu-1}}{2}+\cdots+\frac{\lambda}{\mu}+\frac{1}{\mu+1}
$$

and this inequality is always true for positive $\lambda$ and $\mu$.

Now, since the splitting of the $\delta$-sequence can be made to respect analyticity, and since $\widetilde{\sigma}_{\mu}^{\mu+1}$ is analytic, it follows that $\sigma_{\mu}^{\mu+1}$ is analytic, and we have just seen that

$$
\left\|\sigma_{\mu}^{\mu+1}\right\| \leqq M_{0} \lambda^{\prime \prime+1} h^{\mu+1}(\mu+1) ! .
$$

This reasoning can be repeated, and it completes the proof of the theorem.

In this theorem, we used sufficient conditions. In some cases, it may be possible to see that $K$ exists, without all the assumptions of analyticity, as the following examples will show.

EXAMPLEs. (1) The case where the prolongation vanishes for all sufficiently large $\mu$ as, for example, in the case of the system of equations where the $(n \times n)$-matrix $\left(\partial f^{j} / \partial x^{k}\right)$ belongs to the Lie algebra of a compact lie group. Then there is no problem of convergence, and $K$ will provide a solution for the $D$-Poincaré lemma.

(2) Let $\mathscr{D} f=0$ be the equation

$$
\left\{\begin{array}{l}
\frac{\partial f}{\partial x^{1}}=f \\
\frac{\partial f}{\partial x^{2}}=0 .
\end{array}\right.
$$

(Here $n=2, m=1, l=2, \mu_{0}=1$.) This is a very special example of the constant coefficient case, and here, too, $K$ will actually provide a solution for the $D$-Poincaré lemma, in the following way. Any jet form $\sigma^{\infty}$ belonging to $\Sigma^{\infty}$ admits locally the components

$$
\left\{\begin{aligned}
\sigma_{p_{1}, p_{2}} & =0 \text { if } p_{2} \neq 0, \\
\sigma_{p_{1}, 0} & =\sigma_{0,0}
\end{aligned}\right.
$$

Let $\sigma=\sigma^{\infty} d x^{1}$. We have

$$
\begin{aligned}
K \sigma & =x^{1} \int_{0}^{1} \sum_{l=0}^{\infty}(1-t)^{l} \frac{\left(x^{1}\right)^{l}}{l !} \sigma_{l, 0}(t x) d t=x^{1} \int_{0}^{1} \sigma_{0,0}(t x) \sum_{l=0}^{\infty}(1-t)^{l} \frac{\left(x^{1}\right)^{l}}{l !} d t \\
& =x^{1} \int_{0}^{1} \exp \left\{(1-t) x^{1}\right\} \cdot \sigma_{0,0}(t x) d t .
\end{aligned}
$$

We see that the convergences of the series does not involve here any question of the analyticity of $\sigma$. Unfortunately, this does not generally happen, in the constant coefficient case. Therefore, the operator $K$ cannot be used to prove that the resolution is exact. However, as we 
shall see in the following proposition, the exactness can be used to prove the existence of $K$.

Proposition. If the coefficients of $\mathscr{D}$ are analytic, and if the $D$-Poincare lemma is true, then any $\sigma^{\mu} \in \Sigma^{\mu, r}$, closed under $D^{\mu}$, where $\mu \geqq \mu_{1}, \mu>0$, can be prolonged to an element $\sigma^{\infty} \in \Sigma^{\infty, r}$, closed under $D$ and satisfying (C). Therefore, $K \sigma^{\infty}$ exists.

This lemma does not prove much, of course. It just says that, in some cases, when the $D$-Poincare lemma is true, then the homotopy operator $K$ can be defined. Another problem would be to define a similar operator in general, whether the coefficients of $\mathscr{D}$ are analytic or not, whether the $D$-Poincare lemma is true or not. In the cases where the $D$-Poincaré lemma is not true, we would have to add a "harmonic" term $H$, and write

$$
\sigma=K D \sigma+D K \sigma+H \sigma
$$

as in harmonic theory. We know that this is a difficult problem.

Before proving the proposition, we shall treat a simple example.

ExAMPLE. Let $\mathscr{D} f=\Sigma A_{i}\left(\partial f / \partial x^{i}\right)$ where the $A_{i}$ are $l \times m$ constant matrices, and let us suppose that $\mathscr{D}$ is involutive (i.e., $\mu_{1}=1$ ). In this particular case, we know that the $D^{1}$-Poincare lemma is true.

Let $\sigma^{1}$ be an element of $\Sigma^{1, r}$ with components.

$$
\left\{\begin{array}{l}
\sigma_{-1}^{0} \text { in } \Sigma_{-1}^{0, r}, \\
\sigma_{0}^{1} \text { in } \Sigma_{0}^{1, r}
\end{array}\right.
$$

We suppose that $D^{1} \sigma^{1}=0$. Let $\omega^{2}$ be an element of $\Sigma^{2, r-1}$, with components $\omega_{-1}^{0}, \omega_{0}^{1}, \omega_{1}^{2}$, such that $D^{2} \omega^{2}=\sigma^{1}$, i.e.

$$
\left\{\begin{array}{l}
d \omega_{-1}^{0}-\delta \omega_{0}^{1}=\sigma_{-1}^{0} \\
d \omega_{0}^{1}-\delta \omega_{1}^{2}=\sigma_{0}^{1}
\end{array}\right.
$$

The element $\omega^{2}$ may be prolonged to an element $\omega^{\infty} \in \Sigma^{\infty}$ by adding the zero element of $\Sigma_{3}$, since the $A_{i}$ are constant and the equation contains terms of first order only.

Now let $\sigma^{\infty}=D \omega^{\infty}$. This form has only three components, namely:

$$
\left\{\begin{array}{l}
\sigma_{-1}^{0}=d \omega_{-1}^{0}-\delta \omega_{0}^{1}, \\
\sigma_{0}^{1}=d \omega_{0}^{1}-\delta \omega_{1}^{2}, \\
\sigma_{1}^{2}=d \omega_{1}^{2} .
\end{array}\right.
$$

Therefore, $\sigma^{\infty}$ is a prolongation of $\sigma^{1}$, closed under $D$, and $K \sigma^{\infty}$ exists. This can be generalized to the case where the assumptions of the proposition are satisfied. 
Proof of the proposition. Let $\sigma^{\mu}$ be an element of $\Sigma^{\mu, r}$ such that $D^{\prime \prime} \sigma^{\mu}=0$. By hypothesis, there exists an element $\tau^{\mu+1} \in \Sigma^{\mu+1, r-1}$ such that $D^{\mu+1} \tau^{\mu+1}=\sigma^{\mu}$. An infinite prolongation $\tau^{\infty}$, of $\tau^{\mu+1}$, can be chosen (see Lemma 3) in such a way that $\tau^{\infty}$ satisfies (C). Now, $\sigma^{\infty}=D \tau^{\infty}$ will be an infinite prolongation of $\sigma^{\mu}$, and we just have to prove that $\sigma^{\infty}$ also satisfies (C).

But $D \sigma^{\infty}=d \tau^{\infty}-\delta \tau^{\infty}$. The form $\delta \tau^{\infty}$ obviously satisfies (C) whenever $\tau^{\infty}$ does. The same is true for $d \tau^{\infty}$, although $\tau^{\infty}$ is not necessarily analytic. In fact, $\tau_{\mu+1}^{\mu+2}$, for instance, is a linear combination $\sum_{\nu=0}^{\mu+1} \lambda_{\nu} \tau_{\nu-1}^{\nu}$, as we have seen in the proof of Lemma 3 and, as we have remarked, the coefficients $\lambda_{\nu}$ are analytic. Therefore, $d \tau_{\mu+1}^{\mu+2}$ will be bounded in terms of the $\lambda_{\nu}, \tau_{\nu-1}^{\nu}$, and their first derivatives. The further prolongations will only involve the first derivatives of $\tau_{\nu-1}^{\nu}$, for $0 \leqq \nu \leqq \mu+1$. Therefore, the condition (C) will be satisfied.

Appendix. Homotopy operators in the complex case. Let us consider a complex analytic manifold $M$, of complex dimension $n$. A coordinate $\left(z_{i}\right)$ is chosen in a neighborhood of the origin. Let $\mathscr{D} f$ be the operator

$$
\left\{\begin{array}{l}
\frac{\partial f}{\partial \bar{z}_{1}} \\
\frac{\partial f}{\partial \bar{z}_{n}}
\end{array}\right.
$$

An element $\sigma$ of $\Sigma^{\infty}$ has components $\sigma_{p}$, where the multi-index $p$ is here written $\left(p_{1}, \cdots, p_{n}, p_{\overline{1}}, \cdots, p_{\bar{n}}^{-}\right)$. We have $\sigma_{p}=0$ whenever one at least of the $p_{i}$ is different from 0 .

Now, let $\sigma=\left(\sigma_{p}\right)$ a differential form of bidegree $(r, s)$, belonging to $\Sigma^{\infty, r+s}$. The form $D \sigma$, belonging to $\Sigma^{\infty, r+s+1}$ splits in two parts:

$$
\begin{array}{ll}
D^{\prime} \sigma=\partial \sigma-\delta \sigma, & \text { of bidegree }(r+1, s) \\
D^{\prime \prime} \sigma=\bar{\partial} \sigma, & \text { of bidegree }(r, s+1)
\end{array}
$$

Where $\partial$ means the derivative with respect to the $z_{i}$ 's, and $\bar{\partial}$ the derivative with respect to the $\bar{z}_{i}$ 's.

Both $D^{\prime}$-Poincaré lemma (for $r \geqq 1$ ), and $D^{\prime \prime}$-Poincaré lemma (for $s \geqq 1$ ) are true, but unfortunately our operator $K$ does not give any solution for these lemmas.

In fact, $K \sigma$ (when it converges) is the sum of two forms

$$
\begin{aligned}
& K_{1} \sigma \text {, of bidegree }(r-1, s) \text { if } r \geqq 1 ; 0 \text { if } r=0 \\
& K_{2} \sigma \text {, of bidegree }(r, s-1) \text { if } s \geqq 1 ; 0 \text { if } s=0,
\end{aligned}
$$

and we have the following 


$$
\begin{aligned}
& D^{\prime \prime} K_{1} \sigma+K_{1} D^{\prime \prime} \sigma=0 \\
& D^{\prime} K_{1} \sigma+K_{1} D^{\prime} \sigma+D^{\prime \prime} K_{2} \sigma+K_{2} D^{\prime \prime} \sigma=\sigma \text { if } r+s \geqq 1 \\
& D^{\prime} K_{2} \sigma+K_{2} D^{\prime} \sigma=0 .
\end{aligned}
$$

Nothing more can be said. In other words $K_{1}$ does not solve the $D^{\prime}$-Poincaré lemma, nor $K_{2}$ solves the $D^{\prime \prime}$-Poincaré lemma.

The reason of this failure is obviously that all the variables play symmetrical parts in the definition of $D$-which is therefore ill-adapted to our problem. But we can think of defining two operators $K^{\prime}$ and $K^{\prime \prime}$, for $D^{\prime}$ and $D^{\prime \prime}$, in a way analogous to the one we used to find $K$.

We shall restrict ourselves to analytic jets. We first define the operator $h_{\bar{z}_{i}}$ operating on functions, by

$$
h_{\bar{z}_{i}}(f)=\bar{z}_{i} \int_{0}^{1} f\left(z_{1}, \cdots z_{n}, \bar{z}_{1}, \cdots t \bar{z}_{i}, \cdots \bar{z}_{n}\right) d t,
$$

and we construct $k^{\prime \prime} \omega$, for a form $\omega$ of bidegree $(r, s) s \geqq 1$, in the following standard way.

(1) if $\bar{\partial} \omega=0$, we wite $\omega=d \bar{z}_{\overline{1}} \wedge \alpha_{1}+\beta_{1}$ where $\beta_{1}$ only contains $d z_{1}, \cdots, d z_{n}, d \bar{z}_{2}, \cdots, d \bar{z}_{n}$.

We apply $h_{\bar{z}_{1}}$ to each coefficient of $\alpha_{1}$, thus obtaining a differential form $\gamma_{1}$ such that $\left(\partial \gamma_{1} / \partial \bar{z}_{1}\right)=\alpha_{1}$.

We next consider $\omega-\bar{\partial} \gamma_{1}$, which does not contain $d \bar{z}_{1}$ any more, and the coefficients are holomorphic with respect to $z_{1}$, since $\bar{\partial}\left(\omega-\bar{\partial} \gamma_{1}\right)=0$.

Again, we write $\omega-\bar{\partial} \gamma_{1}=d \bar{z}_{2} \wedge \alpha_{2}+\beta_{2}$, apply $h_{\bar{z}_{2}}$ to each coefficient of $\alpha_{2}$, thus obtaining a form $\gamma_{2}$, and $\omega-\bar{\partial} \gamma_{1}-\bar{\partial} \gamma_{2}$ does not contain $d \bar{z}_{1}$ and $d \bar{z}_{2}$; its coefficients are holomorphie with respect to $z_{1}$ and $z_{2}$.

After $p$ such operations $(p \leqq n)$ there with be no $d \bar{z}_{\bar{i}}$ left. We shall define $k^{\prime \prime} \omega$ to be the sum $\gamma_{1}+\gamma_{2}+\cdots \gamma_{p}$.

(2) if $\bar{\partial} \omega \neq 0$, we define $k^{\prime \prime} \omega=k^{\prime \prime}\left(\omega-k^{\prime \prime} \bar{\partial} \omega\right)$ and this has a meaning since $\bar{\partial}\left(\omega-k^{\prime \prime} \bar{\partial} \omega\right)=0$.

For a differential form $\sigma=\left(\sigma_{p}\right) \in \Sigma^{\infty}$, we define $K^{\prime \prime} \sigma=\left(k^{\prime \prime} \sigma_{p}\right)$. It is now easy to check that

$$
\left\{\begin{aligned}
D^{\prime} K^{\prime \prime}+K^{\prime \prime} D^{\prime} & =0 \\
D^{\prime \prime} K^{\prime \prime}+K^{\prime \prime} D^{\prime \prime} & =I
\end{aligned}\right.
$$

In the same way, for a differential form of bidegree $(r, s) r \geqq 1$, we can define $k^{\prime} \sigma$, thanks to the operators

$$
h_{z_{i}}(f)=z_{i} \int_{0}^{1} f\left(z_{1}, \cdots, t z_{i}, \cdots, z_{n}, \bar{z}_{i}, \cdots, \bar{z}_{n}\right) d t
$$

and $K^{\prime}=k^{\prime} \sum_{1=0}^{\infty}\left(\delta k^{\prime}\right)$ and this operator satisfies

$$
\left\{\begin{aligned}
D^{\prime \prime} K^{\prime}+K^{\prime} D^{\prime \prime} & =0 \\
D^{\prime} K^{\prime}+K^{\prime} D^{\prime} & =I
\end{aligned}\right.
$$


In conclusion, for $s \geqq 1, K^{\prime \prime}$ solves at the same time the $D$-and the $D^{\prime \prime}$-Poincaré lemmas, and for $r \geqq 1, K$ solves at the same time the $D$-and the $D^{\prime}$-Poincaré lemmas.

We wish to express our deep gratitude to Professor D. C. Spencer, who guided and encouraged this work.

\section{REFERENCES}

1. L. Ehrenpreis, V. W. Guillemin and S. Sternberg, On Spencer's estimate for $\delta$ Poincaré, Ann. of Math. 82 (1965), 128-138.

2. V. W. Guillemin and S. Sternberg, An algebraic model of transitive differential geometry, Bull. Amer. Math. Soc. 70 (1964), 16-47.

3. D. G. Quillen, Formal properties of over-determined systems of linear partial differential equations, Harvard Thesis, 1964 (to appear).

4. D. C. Spencer, (a) A type of formal exterior differentiation associated with pseudogroups, Scripta Mathematica 26 (1961), 101-106.

(b) Deformation of structures on manifolds defined by transitive, continuous pseudogroups, I-II, Ann. of Math. 76 (1962), 306-445.

(c) Deformation of structures on manifolds defined by transitive, continuous pseudogroups, III, Ann. of Math. 81 (1965), 389-450.

5. W. J. Sweeney, The $\delta$-Poincaré estimate (to appear).

Received September 9, 1965. This paper was written while the author was engaged part-time on a research project at Stanford University sponsored by the Army Research Office (DA 31-124-ARO(D)-151). 


\section{PACIFIC JOURNAL OF MATHEMATICS}

\section{EDITORS}

H. SAMELSON

Stanford University

Stanford, California

J. P. JANS

University of Washington

Seattle, Washington 98105

\section{J. DugunduI}

University of Southern California Los Angeles, California 90007

RICHARD ARENS

University of California

Los Angeles, California 90024

\section{ASSOCIATE EDITORS}
E. F. BECKENBACH
B. H. NEUMANN
F. WOLF
K. YoSIDA

\section{SUPPORTING INSTITUTIONS}

\author{
UNIVERSITY OF BRITISH COLUMRIA \\ CALIFORNIA INSTITUTE OF TECHNOLOGY \\ UNIVERSITY OF CALIFORNIA \\ MONTANA STATE UNIVERSITY \\ UNIVERSITY OF NEVADA \\ NEW MEXICO STATE UNIVERSITY \\ OREGON STATE UNIVERSITY \\ UNIVERSITY OF OREGON \\ OSAKA UNIVERSITY \\ UNIVERSITY OF SOUTHERN CALIFORNIA
}

\author{
STANFORD UNIVERSITY \\ UNIVERSITY OF TOKYO \\ UNIVERSITY OF UTAH \\ WASHINGTON STATE UNIVERSITY \\ UNIVERSITY OF WASHINGTON \\ AMERICAN MATHEMATICAL SOCIETY \\ CHEVRON RESEARCH CORPORATION \\ TRW SYSTEMS \\ NAVAL ORDNANCE TEST STATION
}

Mathematical papers intended for publication in the Pacific Journal of Mathematics should be typewritten (double spaced). The first paragraph or two must be capable of being used separately as a synopsis of the entire paper. It should not contain references to the bibliography. Manuscripts may be sent to any one of the four editors. All other communications to the editors should be addressed to the managing editor, Richard Arens at the University of California, Los Angeles, California 90024 .

50 reprints per author of each article are furnished free of charge; additional copies may be obtained at cost in multiples of 50 .

The Pacific Journal of Mathematics is published monthly. Effective with Volume 16 the price per volume (3 numbers) is $\$ 8.00$; single issues, $\$ 3.00$. Special price for current issues to individual faculty members of supporting institutions and to individual members of the American Mathematical Society: $\$ 4.00$ per volume; single issues $\$ 1.50$. Back numbers are available.

Subscriptions, orders for back numbers, and changes of address should be sent to Pacific Journal of Mathematics, 103 Highland Boulevard, Berkeley 8, California.

Printed at Kokusai Bunken Insatsusha (International Academic Printing Co., Ltd.), No. 6, 2-chome, Fujimi-cho, Chiyoda-ku, Tokyo, Japan.

\section{PUBLISHED BY PACIFIC JOURNAL OF MATHEMATICS, A NON-PROFIT CORPORATION}

The Supporting Institutions listed above contribute to the cost of publication of this Journal, but they are not owners or publishers and have no responsibility for its content or policies. 


\section{Pacific Journal of Mathematics}

\section{Vol. 21, No. 2 December, 1967}

Arne P. Baartz, The measure algebra of a locally compact semigroup ..... 199

Robert F. Brown, On maps with identical fixed point sets............. 215

C. Buttin, Existence of a homotopy operator for Spencer's sequence in the analytic case ..................................... 219

Henry Werner Davis, An elementary proof that Haar measurable almost periodic functions are continuous ........................ 241

Zeev Ditzian, On asymptotic estimates for kernels of convolution transforms ...................................... 249

Robert E. Edwards, Boundedness principles and Fourier theory ......... 255

John A. Hildebrant, On compact unithetic semigroups ............... 265

Marinus A. Kaashoek and David Clark Lay, On operators whose Fredholm set is the complex plane ............................ 275

Sadao Kató, Canonical domains in several complex variables ........... 279

David Clifford Kay, The ptolemaic inequality in Hilbert geometries.... . . . 293

Joseph D. E. Konhauser, Biorthogonal polynomials suggested by the Laguerre polynomials ............................. 303

Kevin Mor McCrimmon, Macdonald's theorem with inverses .......... 315

Harry Eldon Pickett, Homomorphisms and subalgebras of multialgebras .................................... 327

Richard Dennis Sinkhorn and Paul Joseph Knopp, Concerning nonnegative matrices and doubly stochastic matrices ..............

Erling Stormer, On anti-automorphisms of von Neumann algebras ...

Miyuki Yamada, Regular semi-groups whose idempotents satisfy permutation identities .......................... 